\title{
Constraints on the Astrophysical Nature of r-Process Nucleosynthesis Sites from Inhomogeneous Chemical Evolution Models
}

\author{
Dominik Argast ${ }^{1,2,3}$ and Markus Samland ${ }^{2}$ \\ ${ }^{1}$ Institut für Physik Universität Basel, CH 4056 Basel, Switzerland \\ 2 Astronomisches Institut Universität Basel, CH 4102 Binningen, Switzerland \\ ${ }^{3}$ E-mail: argast@quasar.physik.unibas.ch
}

Received 2003 September 28, accepted 2004 March 1

\begin{abstract}
We present a detailed inhomogeneous chemical evolution study that considers for the first-time neutron star mergers as major r-process sources, and compare this scenario with the ones in which lower-mass (in the range $8-10 \mathrm{M}_{\odot}$ ) or higher-mass core-collapse supernovae (with masses $\geq 20 \mathrm{M}_{\odot}$ ) act as dominant r-process sites. We conclude that it is not possible at present to distinguish between the lower-mass and higher-mass supernovae scenarios within the framework of inhomogeneous chemical evolution. However, neutron-star mergers seem to be ruled out as the dominant r-process source, since their low rates of occurrence would lead to r-process enrichment that is not consistent with observations.
\end{abstract}

Keywords: nuclear reactions, nucleosynthesis, abundances — stars: abundances — ISM: abundances — Galaxy: abundances — Galaxy: evolution — Galaxy: halo

\section{Introduction}

Heavy elements beyond the iron peak are formed in part by r-process nucleosynthesis, i.e. the rapid capture of neutrons on seed nuclei. Yet although the physical requirements for the occurrence of r-process nucleosynthesis are well understood, the astrophysical nature of the dominant $r$-process site is still unknown. The abundance pattern of neutron capture elements heavier than $\mathrm{Ba}(Z \geq 56)$ in ultra metal-poor stars matches the scaled solar system r-process abundances remarkably well. This suggests that the synthesis of r-process elements started early in Galactic evolution and that the r-process is robust for elements heavier than $\mathrm{Ba}$, i.e. it originates from a single astrophysical site or at least occurs under well defined physical conditions (e.g. Sneden et al. 2000; Westin et al. 2000; Cowan et al. 2002). On the other hand, lighter neutroncapture elements $(30<Z<56)$ in ultra metal-poor halo stars show significant deviations from the scaled solar system r-process abundance curve, indicating the possible existence of a second r-process source. However, the evidence that the r-process beyond $\mathrm{Ba}$ is robust is very convincing, and we will use this property in the following to gain some insight into the enrichment of the Galaxy with r-process elements beyond $\mathrm{Ba}$.

A number of possible astrophysical sites responsible for the robust $r$-process were put forth in the past, most of them linked to the violent death of massive stars in supernova events. Wheeler et al. (1998) suggested that, during the prompt explosion of a massive star in the range $8-10 \mathrm{M}_{\odot}$, physical conditions in the innermost mass layers are sufficiently extreme for the r-process to work, and that the amount of r-process matter ejected may be consistent with observed Galactic r-process abundances. However, there are major objections to the prompt explosion mechanism from detailed studies by Bruenn (1989a, $1989 b$ ). On the other hand, neutrino-driven winds from nascent neutron stars were also proposed as a promising site for r-process nucleosynthesis (e.g. Woosley \& Hoffman 1992; Takahashi et al. 1994; Woosley et al. 1994; Qian \& Woosley 1996; Thompson et al. 2001; Wanajo et al. 2001; Terasawa et al. 2002). However, r-process yields consistent with observed r-process abundances in stars may be obtained in this scenario only for extreme assumptions such as massive neutron stars of $2 \mathrm{M}_{\odot}$ or more, which makes this an unlikely scenario. Recently, Freiburghaus et al. (1999) presented for the first time detailed r-process calculations for neutron star mergers (NSMs). Coalescing neutron stars potentially can provide in a natural way the large neutron fluxes required for the build up of heavy elements through rapid neutron capture. This scenario was subsequently explored and refined by Rosswog et al. (1999, 2000). However, Qian (2000) argued that if NSMs were a major r-process source, the low coalescence rate of binary compact objects would prevent any correlation between abundances of r-process elements and iron, which is clearly in disagreement with observed r-process abundances in stars more metal-rich than $[\mathrm{Fe} / \mathrm{H}] \sim-2.5$. In addition, the large amount of r-process ejecta from NSMs would lead to r-process abundances in metal-poor halo stars that would be too large compared to observations.

The purpose of this work is twofold. First, neutron star mergers are included as major r-process sites in detailed inhomogeneous chemical evolution calculations for the 
first time. Second, we compare the r-process enrichment of the ISM under the assumption that NSMs, lower-mass $\mathrm{SNe}$ II or higher-mass SNe II are the dominant r-process sites in the framework of inhomogeneous chemical evolution. The stochastic chemical evolution model used in this work was described in Argast et al. (2000).

\section{Treatment of r-Process Sources in the Model}

An ideal tracer of the r-process enrichment of the ISM is the pure r-process element europium. Unfortunately, only a small sample of Eu abundances at very low metallicities (i.e. $[\mathrm{Fe} / \mathrm{H}] \leq-2.5$ ) are available to date. In order to trace the r-process enrichment at lower metallicities, the well-studied element barium is also used in this investigation. Note that $\mathrm{Ba}$ abundances in stars are dominated by the s-process, and only $\approx 15 \%$ of the solar Ba abundance was formed by rapid neutron capture (Burris et al. 2000). However, the r-process fraction $\left[\mathrm{Ba}^{\mathrm{r}} / \mathrm{Fe}\right]$ of $\mathrm{Ba}$ abundances can be computed by removing the s-process contribution to $\mathrm{Ba}$ in stars with $[\mathrm{Fe} / \mathrm{H}]>-2.5$ if Eu abundances have also been determined (cf. Burris et al. 2000; but see also Johnson \& Bolte 2001). For stars with metallicities $[\mathrm{Fe} / \mathrm{H}]<-2.5$, it is assumed that the whole Ba inventory is of pure r-process origin. Care has been taken to remove known carbon stars from our sample. Such stars mostly show unusually large $\mathrm{Ba}$ abundances, which are thought to originate from mass transfer of s-process enriched matter in binary systems.

In the following, r-process yields of Eu and $\mathrm{Ba}$ are estimated under the assumption of a robust r-process for nuclei more massive than $\mathrm{Ba}$, i.e. only one source is responsible for the enrichment of the ISM with r-process elements beyond $Z=56$. The yields were chosen in such a way that the whole range of r-process abundances and its average in metal-poor halo stars are reproduced in the model. Note that r-process yields have in essence been treated as free parameters and are tuned to match the observations. There are no theoretical r-process yields available to date. Furthermore, the progenitor mass dependence of Fe yields, which is essential to determine the maximal range of $[\mathrm{Eu} / \mathrm{Fe}]$ and $\left[\mathrm{Ba}^{\mathrm{r}} / \mathrm{Fe}\right]$ ratios, is poorly understood (Argast et al. 2002).

\section{1 r-Process Yields from Core-Collapse SNe}

Lower mass core-collapse $\mathrm{SNe}\left(8-10 \mathrm{M}_{\odot}\right)$ are suspected to be major r-process sources without contributing much of the iron-peak elements to the enrichment of the ISM (Wheeler et al. 1998). In model SN810, r-process nucleosynthesis is assumed to occur in the mass range $8-10 \mathrm{M}_{\odot}$ with constant $\mathrm{Ba}$ and $\mathrm{Eu}$ yields over the whole mass range. The yields are then deduced from the average $[\mathrm{Eu} / \mathrm{Fe}]$ and $\left[\mathrm{Ba}^{\mathrm{r}} / \mathrm{Fe}\right]$ ratios (both $\approx 0.5$ ) of metal-poor halo stars. Furthermore, we assume that the amount of $\alpha$ and iron peak elements synthesised in these SN II events is negligible (Hillebrandt et al. 1984).

Nucleosynthesis of r-process elements might also occur in neutrino driven winds or jets from nascent neutron stars during the delayed explosion of high-mass stars
Table 1. Adopted $\mathrm{Ba}\left(Y_{\mathrm{Ba}}(m)\right)$ and $\mathrm{Eu}\left(Y_{\mathrm{Eu}}(m)\right)$ yields and ejected r-process matter $\left(M_{r}\right)$ as function of progenitor mass $m$ of models SN810 and SN2050

Yields in the mass range $8-10 \mathrm{M}_{\odot}$ are assumed to be constant (SN810). In model SN2050, a rapid decline in the mass range

$20-28 \mathrm{M}_{\odot}$ is assumed. Progenitors with masses $28-50 \mathrm{M}_{\odot}$ all have the same yield

\begin{tabular}{clll}
\hline$m$ & $Y_{\mathrm{Ba}}(m)\left[\mathrm{M}_{\odot}\right]$ & $Y_{\mathrm{Eu}}(m)\left[\mathrm{M}_{\odot}\right]$ & $M_{r}\left[\mathrm{M}_{\odot}\right]$ \\
\hline $8^{a}$ & $5.3 \times 10^{-7}$ & $8.3 \times 10^{-8}$ & $6.9 \times 10^{-6}$ \\
10 & $5.3 \times 10^{-7}$ & $8.3 \times 10^{-8}$ & $6.9 \times 10^{-6}$ \\
$20^{b}$ & $1.1 \times 10^{-5}$ & $1.8 \times 10^{-6}$ & $1.5 \times 10^{-4}$ \\
21 & $3.3 \times 10^{-6}$ & $5.1 \times 10^{-7}$ & $4.2 \times 10^{-5}$ \\
22 & $7.9 \times 10^{-7}$ & $1.2 \times 10^{-7}$ & $1.0 \times 10^{-5}$ \\
23 & $2.5 \times 10^{-7}$ & $3.8 \times 10^{-8}$ & $3.2 \times 10^{-6}$ \\
24 & $9.2 \times 10^{-8}$ & $1.4 \times 10^{-8}$ & $1.2 \times 10^{-6}$ \\
25 & $4.8 \times 10^{-8}$ & $7.5 \times 10^{-9}$ & $6.2 \times 10^{-7}$ \\
26 & $2.7 \times 10^{-8}$ & $4.3 \times 10^{-9}$ & $3.6 \times 10^{-7}$ \\
27 & $1.8 \times 10^{-8}$ & $2.7 \times 10^{-9}$ & $2.3 \times 10^{-7}$ \\
28 & $1.1 \times 10^{-8}$ & $1.7 \times 10^{-9}$ & $1.4 \times 10^{-7}$ \\
50 & $1.1 \times 10^{-8}$ & $1.7 \times 10^{-9}$ & $1.4 \times 10^{-7}$ \\
\hline
\end{tabular}

Remarks: ${ }^{a}$ Model SN810. $\quad{ }^{b}$ Model SN2050.

with masses $\geq 20 \mathrm{M}_{\odot}$ (e.g. Woosley \& Hoffman 1992; Takahashi et al. 1994; Woosley et al. 1994; Qian \& Woosley 1996; Thompson et al. 2001; Nagataki 2001; Wanajo et al. 2001, 2002; Terasawa et al. 2002). As an alternative to model SN810, we also calculated the chemical evolution of the ISM with r-process yields from SNe II in the mass range $20-50 \mathrm{M}_{\odot}$ (model SN2050). The r-process yield of the $20 \mathrm{M}_{\odot}$ is chosen so that it reproduces the largest $[\mathrm{Eu} / \mathrm{Fe}]$ and $\left[\mathrm{Ba}^{\mathrm{r}} / \mathrm{Fe}\right]$ ratios of metal-poor halo stars observed. Since this requires a large amount of ejected r-process matter, the yields have to decrease sharply with increasing progenitor mass to account for the average $[\mathrm{Eu} / \mathrm{Fe}]$ and $\left[\mathrm{Ba}^{\mathrm{r}} / \mathrm{Fe}\right]$ abundance ratios of metalpoor halo stars.

Table 1 lists our Ba yields $Y_{\mathrm{Ba}}(m)$, Eu yields $Y_{\mathrm{Eu}}(m)$, and total ejected r-process matter $M_{\mathrm{r}}$ as functions of progenitor mass $m$ of models SN810 and SN2050.

\section{2 r-Process Yields from Neutron Star Mergers}

Another major source of r-process elements might be neutron star mergers (Freiburghaus et al. 1999; Rosswog et al. 1999, 2000). Constraints on the properties of the Galactic NSM population, however, are controversial. Coalescence timescales for neutron star mergers are typically estimated to be of the order 100-1000 Myr (e.g. Portegies Zwart \& Yungel'son 1998; Fryer et al. 1999). Recently, Belczynski et al. (2002) suggested a dominant population of short lived neutron star binaries with merger times less than $1 \mathrm{Myr}$. In view of these uncertainties, we adopt $1 \mathrm{Myr}$ as the coalescence timescale, which favours the NSM scenario (cf. Section 3.2). Galactic NSM rates are estimated by van den Heuvel \& Lorimer (1996) to approximately $8 \times 10^{-6} \mathrm{yr}^{-1}$, Kalogera \& Lorimer (2000) give an upper limit of $\left(7.5 \times 10^{-7}-1.5 \times 10^{-5}\right) \mathrm{yr}^{-1}$, whereas Belczynski et al. (2002) obtain rates in the range $\left(10^{-6}-3 \times 10^{-4}\right) \mathrm{yr}^{-1}$. The amount $M_{\mathrm{r}}$ of r-process 
Table 2. Constraints on the Galactic NSM rate $f_{\text {NSM }}$ and ejected r-process matter $M_{\mathbf{r}}$

\begin{tabular}{ll}
\hline Reference & $f_{\mathrm{NSM}}\left[\mathrm{yr}^{-1}\right]$ \\
van den Heuvel \& Lorimer (1996) & $8 \times 10^{-6}$ \\
Kalogera \& Lorimer (2000) & $\leq\left(7.5 \times 10^{-7}-1.5 \times 10^{-5}\right)$ \\
Belczynski et al. (2002) & $10^{-6}-3 \times 10^{-4}$ \\
Reference & $M_{\mathrm{r}}\left[\mathrm{M}_{\odot}\right]$ \\
Rosswog et al. (1999) & $10^{-3}-10^{-2}$ \\
Oechslin et al. (2002) & $\geq\left(5 \times 10^{-5}-2 \times 10^{-4}\right)$ \\
\hline
\end{tabular}

matter ejected in a NSM event is of the order of $10^{-3}$ $10^{-2} \mathrm{M}_{\odot}$, depending on the initial configuration of the binary system (Rosswog et al. 1999). Taking general relativistic effects into account, Oechslin et al. (2002) find $5 \times 10^{-5}-2 \times 10^{-4} \mathrm{M}_{\odot}$ as the lower limit for $M_{\mathrm{r}}$. All constraints are again listed in Table 2.

To account for these large uncertainties, four models, with average NSM rates of $2 \times 10^{-3} \mathrm{yr}^{-1}, 2 \times 10^{-4} \mathrm{yr}^{-1}$, $2 \times 10^{-5} \mathrm{yr}^{-1}$, and $2 \times 10^{-6} \mathrm{yr}^{-1}$, have been calculated. Note that the actual NSM rate directly depends on the star formation rate, which determines the frequency of NSM progenitors, and is not constant with time. The amount of ejected r-process matter is $10^{-4} \mathrm{M}_{\odot}, 10^{-3} \mathrm{M}_{\odot}, 10^{-2} \mathrm{M}_{\odot}$, and $10^{-1} \mathrm{M}_{\odot}$, respectively. The NSM rate and the amount of ejected r-process matter are tightly correlated, since the total amount of r-process matter in the Galaxy $\left(\approx 10^{4} \mathrm{M}_{\odot}\right.$, Wallerstein et al. 1997) has to be reproduced. Thus, higher NSM rates require that less r-process matter is ejected in each event, and vice versa.

\section{Enrichment of the ISM with r-Process Elements}

\subsection{SN II as Dominant r-Process Sites}

The results of models SN810 and SN2050 are shown in Figures 1 and 2, respectively. The figures show the evolution of $[\mathrm{Eu} / \mathrm{Fe}]$ and $\left[\mathrm{Ba}^{\mathrm{r}} / \mathrm{Fe}\right]$ as functions of metallicity $[\mathrm{Fe} / \mathrm{H}]$. Note that the $\left[\mathrm{Ba}^{\mathrm{r}} / \mathrm{Fe}\right]$ plots only show the r-process contribution to the total $\mathrm{Ba}$ abundances of halo stars. The evolution of r-process elements shown in Figures 1 and 2 are qualitatively very similar. At very low metallicities $([\mathrm{Fe} / \mathrm{H}] \leq-2.5)$, a large scatter in abundances of model stars is visible. This scatter is due to chemical inhomogeneities in the early ISM (cf. Argast et al. 2000). The scatter decreases as the mixing of the ISM improves and finally reaches the initial mass function averaged mean. At this stage, the ISM can be considered well mixed and the further evolution is comparable to the one of classic chemical evolution models. In the following, we point out some important features of the ISM enrichment resulting from models SN810 and SN2050.

1. In both models, r-process elements appear very early in the enrichment of the ISM. Some model stars with r-process abundances exist even at metallicities $[\mathrm{Fe} / \mathrm{H}] \leq-4$, which is in agreement with observed $\mathrm{Ba}$ abundances in ultra metal-poor stars.
2. For metallicities $[\mathrm{Fe} / \mathrm{H}] \geq-2$, the scatter in $[\mathrm{Eu} / \mathrm{Fe}]$ and $\left[\mathrm{Ba}^{\mathrm{r}} / \mathrm{Fe}\right]$ abundances of model stars is comparable to that seen in observations.

3. Model SN810 fails to reproduce the two metal-poor stars with the highest Eu abundances $([\mathrm{Eu} / \mathrm{Fe}]>+2.0$ at $[\mathrm{Fe} / \mathrm{H}] \approx-2.5)$. The existence of such ultra r-process enhanced stars might pose a serious problem for the $\mathrm{SN}$ scenario, since an unusual large amount of ejected r-process matter $\left(\geq 10^{-4} \mathrm{M}_{\odot}\right)$ is required to reproduce these observations in chemical evolution models (model SN2050, Figure 2).

4. The models also predict some stars with very low $[\mathrm{Eu} / \mathrm{Fe}]$ and $\left[\mathrm{Ba}^{\mathrm{r}} / \mathrm{Fe}\right]$ ratios at $[\mathrm{Fe} / \mathrm{H}] \leq-2$, which are not observed to date. However, if stars with $[\mathrm{r} / \mathrm{Fe}] \leq-1$ exist in the Galactic halo, their Eu and Ba lines may be too weak to be detectable (cf. observational limits quoted in Travaglio et al. 2001).

In summary, core-collapse $\mathrm{SNe}$ seem to be a valid source of r-process elements from the point of view of chemical evolution, since the enrichment of the ISM in the cases discussed above is in qualitative, if not necessarily quantitative, agreement with observations.

\subsection{NSMs as Dominant r-Process Sites}

For the NSM scenario, four model-runs were carried out, assuming NSM rates ranging from $2 \times 10^{-3} \mathrm{yr}^{-1}$ to $2 \times 10^{-6} \mathrm{yr}^{-1}$ and a coalescence timescale of $1 \mathrm{Myr}$. A condensed overview of all models displaying the evolution of $\left[\mathrm{Ba}^{\mathrm{r}} / \mathrm{Fe}\right]$ is shown in Figure 3. The evolution of r-process abundances is strikingly different from the case in which r-process nucleosynthesis occurs in SNe II. All models with NSMs as r-process sources fail to reproduce observations.

The upper left panel of Figure 3 gives the best fit to observations in the NSM case, although model stars with r-process contributions at $[\mathrm{Fe} / \mathrm{H}]<-3$ are still somewhat scarce. However, a comparison with Table 2 shows that the adopted NSM rate of $2 \times 10^{-3} \mathrm{yr}^{-1}$ in this case is one order of magnitude larger than the estimate by Belczynski et al. (2002), whereas the amount of ejected r-process matter of $10^{-4} \mathrm{M}_{\odot}$ is of the same order as the lower limit given by Oechslin et al. (2002). Thus, it is not consistent with the present estimates of the Galactic NSM rate.

As a representative example of the remaining models with consistent NSM rates, the model shown in the upper right panel of Figure 3 is discussed in the following. It was calculated with a NSM rate of $2 \times 10^{-4} \mathrm{yr}^{-1}$ and a total of $10^{-3} \mathrm{M}_{\odot}$ of ejected r-process matter. The following qualitative differences are immediately visible, if the upper right panel of Figure 3 is compared to Figures 1 and 2:

1. r-Process nuclei appear at the earliest around $[\mathrm{Fe} / \mathrm{H}] \approx-2.5$, whereas r-process elements such as $\mathrm{Eu}$ are observed at $[\mathrm{Fe} / \mathrm{H}]=-3$ and probably even down to $[\mathrm{Fe} / \mathrm{H}]=-4$ in the case of $\mathrm{Ba}$. The reason for this late injection of r-process matter in the model is the low NSM rate. 

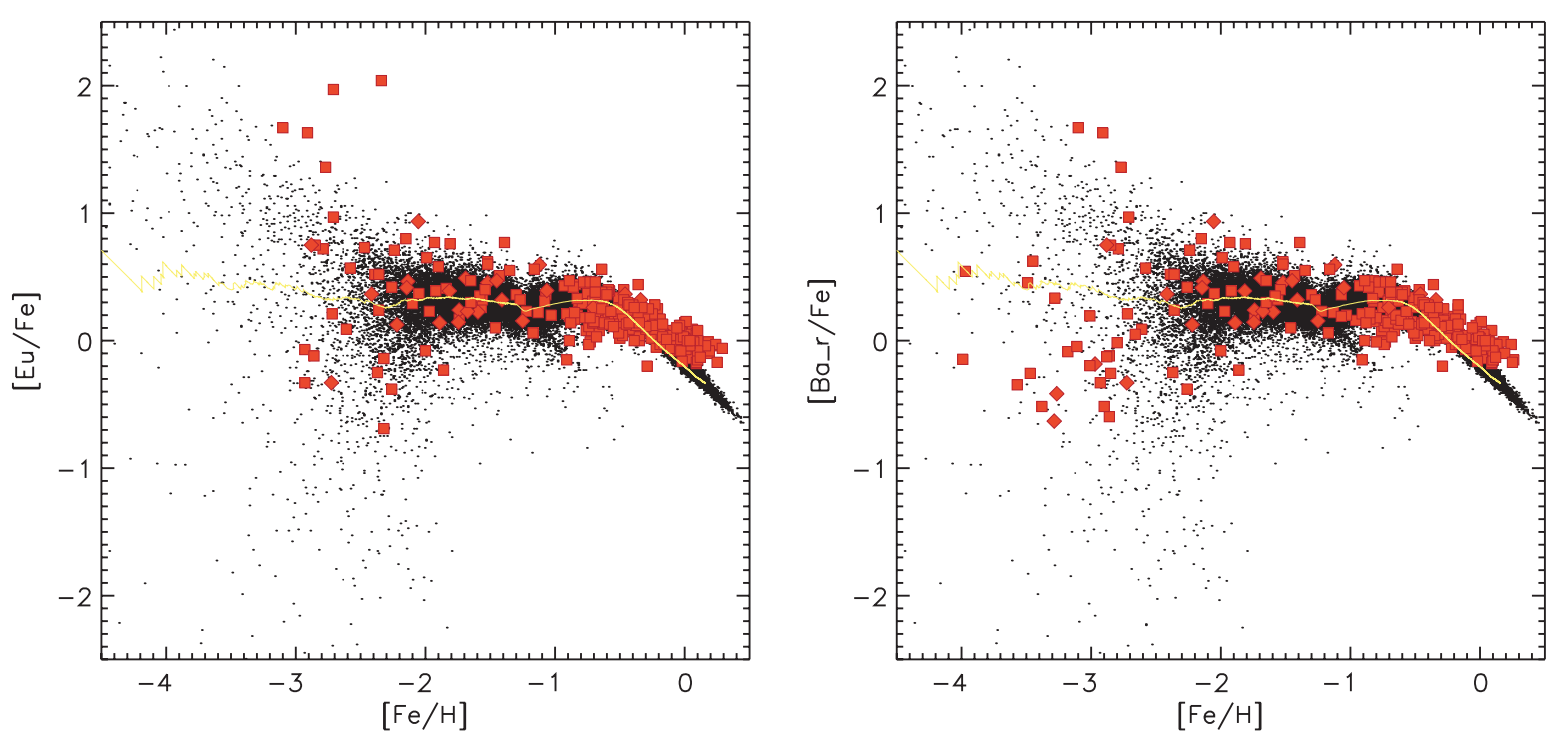

Figure 1 Evolution of $[\mathrm{Eu} / \mathrm{Fe}]$ and $\left[\mathrm{Ba}^{\mathrm{r}} / \mathrm{Fe}\right]$ abundances as functions of metallicity $[\mathrm{Fe} / \mathrm{H}]$. Lower-mass SNe II (Model SN810) are assumed to be the dominant r-process sources. Black dots denote model stars; observations are marked by filled squares and diamonds. Average ISM abundances are marked by a continuous line.
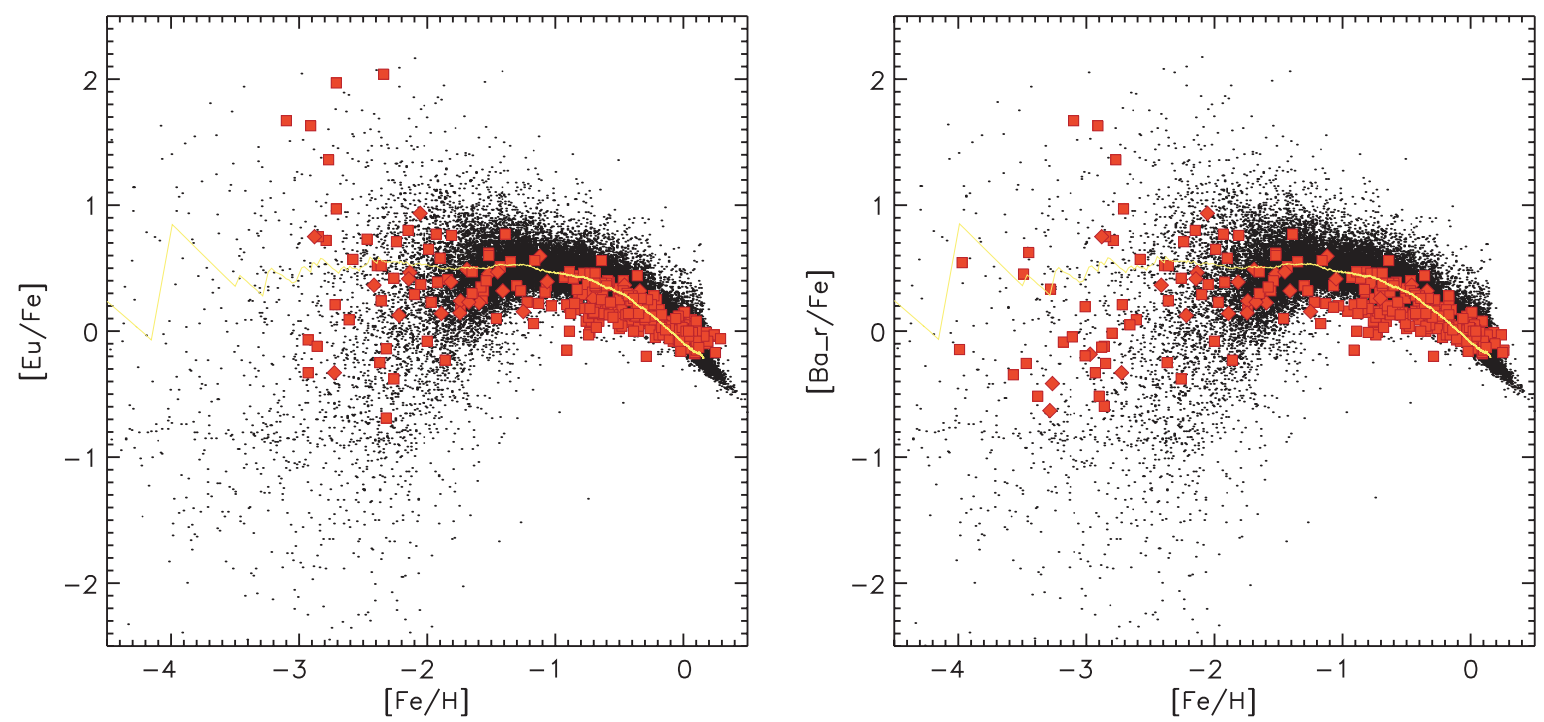

Figure 2 Evolution of $[\mathrm{Eu} / \mathrm{Fe}]$ and $\left[\mathrm{Ba}^{\mathrm{r}} / \mathrm{Fe}\right]$ abundances as functions of metallicity $[\mathrm{Fe} / \mathrm{H}]$. Higher-mass SNe II (Model SN2050) are assumed to be the dominant r-process sources. Symbols are as in Figure 1.

2. There is a prominent tail of model stars with very low $\left[\mathrm{Ba}^{\mathrm{r}} / \mathrm{Fe}\right]$ ratios at $[\mathrm{Fe} / \mathrm{H}]=-2$. Such model stars are also present in Figures 1 and 2. In this case, however, the tail does not develop until $[\mathrm{Fe} / \mathrm{H}] \geq-2$. Since the tail develops at higher metallicities, it cannot be dismissed as unobservable.

3. Even late in the enrichment of the ISM $([\mathrm{Fe} / \mathrm{H}] \geq-1)$, the scatter in possible $[\mathrm{r} / \mathrm{Fe}]$ ratios is of the order $1.5-2.0 \mathrm{dex}$, whereas observations of $[\mathrm{Eu} / \mathrm{Fe}]$ and $\left[\mathrm{Ba}{ }^{\mathrm{r}} / \mathrm{Fe}\right]$ abundances show a scatter of approximately $0.2-0.3$ dex.

These aspects strongly argue against NSMs as the dominant $\mathrm{r}$-process source, especially since the parameters used for the model in discussion are at the upper limit set by theoretical and observational constraints. Lower NSM rates, and consequently larger ejecta masses, only aggravate the problems mentioned above. The dramatic changes in the distribution of r-process abundances occurring with decreasing NSM rate are clearly visible in the sequence of plots in Figure 3. The NSM rates adopted in the models decrease from left to right and top to bottom by one order of magnitude for each panel, i.e. from $2 \times 10^{-3} \mathrm{yr}^{-1}$ for the upper left panel down to $2 \times 10^{-6} \mathrm{yr}^{-1}$ for the lower right panel.

In the upper left panels of Figure 3 (NSM rate $\left.2 \times 10^{-3} \mathrm{yr}^{-1}\right)$, a few model stars with $\mathrm{r}$-process abundances first appear around $[\mathrm{Fe} / \mathrm{H}] \approx-3.5$, in contrast 

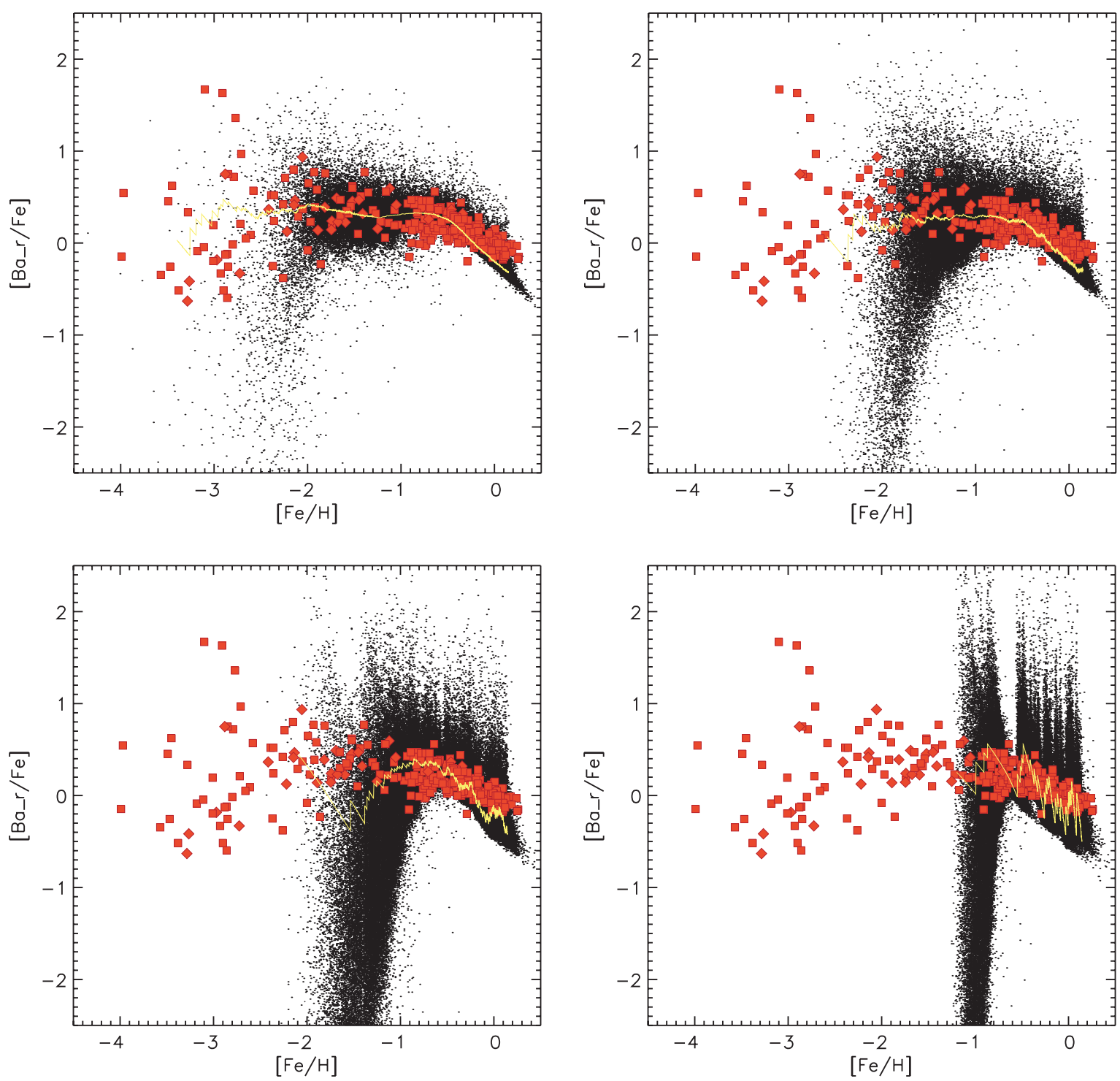

Figure $3\left[\mathrm{Ba}^{\mathrm{r}} / \mathrm{Fe}\right]$ versus $[\mathrm{Fe} / \mathrm{H}]$ for the NSM rates $2 \times 10^{-3} \mathrm{yr}^{-1}, 2 \times 10^{-4} \mathrm{yr}^{-1}, 2 \times 10^{-5} \mathrm{yr}^{-1}$, and $2 \times 10^{-6} \mathrm{yr}^{-1}$ (from left to right and top to bottom). The coalescence timescale adopted in these cases is $10^{6} \mathrm{yr}$. Symbols are as in Figure 1.

to $[\mathrm{Fe} / \mathrm{H}] \approx-1$ in the panels at the lower right (NSM rate $\left.2 \times 10^{-6} \mathrm{yr}^{-1}\right)$. Simultaneously, the scatter in $\left[\mathrm{Ba}^{\mathrm{r}} / \mathrm{Fe}\right]$ and $[\mathrm{Eu} / \mathrm{Fe}]$ at solar metallicity, which is of the same order as that observed in the upper left panel, increases to almost 3 dex in the lower right panel, clearly not consistent with observations. Furthermore, the tail of model stars with low $[\mathrm{r} / \mathrm{Fe}]$ abundances becomes more pronounced and concentrated and is shifted to higher metallicities.

\section{Conclusions}

In this work, we study the enrichment of the ISM with r-process elements in the framework of inhomogeneous chemical evolution. We present a detailed comparison of the impact of lower-mass SNe II (8-10 $\left.\mathrm{M}_{\odot}\right)$, higher-mass $\mathrm{SNe}$ II $\left(\geq 20 \mathrm{M}_{\odot}\right)$, and NSMs as major r-process sites on the enrichment history of the early Galaxy.

In both SNe II scenarios, the model results are qualitative similar to the pattern of r-process abundances in ultra metal-poor stars. However, we conclude that, due to the large uncertainties inherent in the progenitor mass dependence of iron yields of SNe II (Argast et al. 2000, 2002), it is not possible to clearly rule out either lowermass SNe II or higher-mass SNe II as dominant r-process sites from the point of view of inhomogeneous chemical evolution. Additional uncertainties are introduced by the fact that reliable r-process yields from SNe II are unavailable as yet. Here, they were deduced in such a way that the average $[\mathrm{r} / \mathrm{Fe}]$ abundances in metal-poor halo stars are reproduced. Clearly, iron and r-process yields and their dependence on progenitor mass from self-consistent corecollapse SN calculations are required before any decisive conclusion can be reached.

On the other hand, NSMs seem to be ruled out as major r-process sources for the following reasons:

1. Estimates of the Galactic NSM rate are two to three orders of magnitude lower than estimates of the 
Galactic SNe II rate. Thus, the injection of r-process nuclei into the ISM by NSMs would occur late during Galaxy formation $([\mathrm{Fe} / \mathrm{H}] \approx-2.5)$, whereas r-process elements are already observed at $[\mathrm{Fe} / \mathrm{H}]=-3$ and probably even at $[\mathrm{Fe} / \mathrm{H}]=-4$.

2. The late injection of r-process elements furthermore leads to prominent tails in the distribution of r-process abundances down to very low $[\mathrm{r} / \mathrm{Fe}]$ ratios at $[\mathrm{Fe} / \mathrm{H}] \geq-2$, which are seemingly not consistent with observations.

3. Since NSMs occur at a lower rate than SNe II, their r-process yield has to be about two orders of magnitude higher than the r-process yield of typical SNe II. Due to this large r-process yield, considerable chemical inhomogeneities in the ISM are expected to be present even at solar metallicity. The scatter in $[\mathrm{r} / \mathrm{Fe}]$ is predicted to be of the order 2.0-2.5 dex, whereas a scatter of only $0.2-0.3$ dex is observed.

Thus, we conclude that the exact astrophysical nature of r-process sites still remains a mystery, since it is not possible to clearly distinguish between neutron-capture element abundance patterns resulting from lower-mass $\mathrm{SNe}$ II $\left(8-10 \mathrm{M}_{\odot}\right)$ and those from higher-mass SNe II $\left(\geq 20 \mathrm{M}_{\odot}\right)$ in the framework of inhomogeneous chemical evolution. However, the present investigation suggests that core-collapse $\mathrm{SNe}$ are much more likely to be the dominant $\mathrm{r}$-process sites than coalescing neutron star binaries, which at least reduces the list of possible major contributors of r-process nuclei to the enrichment of the ISM. However, it remains to be seen how SNe II can actually produce the required r-process yields.

An extended discussion of these results can be found in Argast et al. (2004).

\section{Acknowledgements}

The authors thank the anonymous referees for their constructive remarks which helped to improve this paper.

\section{References}

Argast, D., Samland, M., Gerhard, O. E., \& Thielemann, F.-K. 2000, A\&A, 356, 873
Argast, D., Samland, M., Thielemann, F.-K., \& Gerhard, O. E. 2002, A\&A, 388, 842

Argast, D., Samland, M., Thielemann, F.-K., \& Qian, Y. Z. 2004, A\&A, 416, 997

Belczynski, K., Kalogera, V., \& Bulik, T. 2002, ApJ, 572, 407

Bruenn, S. W. 1989a, ApJ, 340, 955

Bruenn, S. W. 1989b, ApJ, 341, 385

Burris, D. L., Pilachowski, C. A., Armandroff, T. E., Sneden, C., Cowan, J. J., \& Roe, H. 2000, ApJ, 544, 302

Cowan, J. J., et al. 2002, ApJ, 572, 861

Freiburghaus, C., Rosswog, S., \& Thielemann, F.-K. 1999, ApJ, 525, L121

Fryer, C. L., Woosley, S. E., \& Hartmann, D. H. 1999, ApJ, 526, 152 Hillebrandt, W., Nomoto, K., \& Wolff, R. G. 1984, A\&A, 133, 175

Johnson, J. A., \& Bolte, M. 2001, ApJ, 554, 888

Kalogera, V., \& Lorimer, D. R. 2000, ApJ, 530, 890

Nagataki, S. 2001, ApJ, 551, 429

Oechslin, R., Rosswog, S., \& Thielemann, F. K. 2002, PhRvD, 65, 103005

Portegies Zwart, S. F., \& Yungel'son, L. R. 1998, A\&A, 332, 173

Qian, Y.-Z. 2000, ApJ, 534, L67

Qian, Y.-Z., \& Woosley, S. E. 1996, ApJ, 471, 331

Rosswog, S., Liebendörfer, M., Thielemann, F.-K., Davies, M. B., Benz, W., \& Piran, T. 1999, A\&A, 341, 499

Rosswog, S., Davies, M. B., Thielemann, F.-K., \& Piran, T. 2000, A\&A, 360, 171

Sneden, C., Cowan, J. J., Ivans, I. I., Fuller, G. M., Burles, S., Beers, T. C., \& Lawler, J. E. 2000, ApJ, 533, L139

Takahashi, K., Witti, J., \& Janka, H.-T. 1994, A\&A, 286, 857

Terasawa, M., Sumiyoshi, K., Yamada, S., Suzuki, H., \& Kajino, T. 2002, ApJ, 578, L137

Thompson, T. A., Burrows, A., \& Bradley, S. M. 2001, ApJ, 562, 887

Travaglio, C., Galli, D., \& Burkert, A. 2001, ApJ, 547, 217

van den Heuvel, E., \& Lorimer, D. 1996, MNRAS, 283, L37

Wallerstein, G., et al. 1997, RvMP, 69, 995

Wanajo, S., Toshitaka, K., Mathews, G. J., \& Otsuki, K. 2001, ApJ, 554,578

Wanajo, S., Itoh, N., Ishimaru, Y., Nozawa, S., \& Beers, T. C. 2002, ApJ, 577, 853

Westin, J., Sneden, C., Gustafsson, B., \& Cowan, J. J. 2000, ApJ, 530,783

Wheeler, J. C., Cowan, J. J., \& Hillebrandt, W. 1998, ApJ, 493, L101

Woosley, S. E., \& Hoffman, R. D. 1992, ApJ, 395, 202

Woosley, S. E., Wilson, J. R., Mathews, G. J., Hoffman, R. D., \& Meyer, B. S. 1994, ApJ, 433, 229 